Ann. Génét. Sél. anim., I978, 10 (I), I25-I29.

\title{
Note
}

\section{Déséquilibres de linkage entre les locus Hal (Hyperthermie maligne) PHI et 6-PGD dans deux lignées Piétrain}

\author{
G. GUÉRIN, L. OLLIVIER (*) et P. SELLIER (*)
}

avec la collaboration technique de Marie-Reine LANGLors et P. Dando

\author{
Laboratoive de Génétique biochimique,

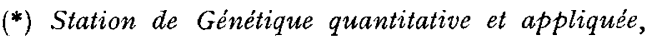 \\ Centre national de Recherches zootechniques, I.N.R.A. \\ $7835^{\circ}$ Jouy-en-Josas, France
}

\begin{abstract}
Résumé
Un échantillon de $25^{\circ}$ pores de Piétrain provenant de deux lignées sélectionnées depuis 3 générations, l'une (A) pour la vitesse de croissance et l'épaisseur de lard et l'autre (B) pour l'hypertrophie musculaire, a été soumis à un test à l'halothane et les génotypes ont été déterminés pour deux enzymes du globule rouge (PHI et 6-PGD). La présence parmi les animaux sensibles à l'halothane de i I sujets hétérozygotes au locus de la PHI démontre formellement que le gène de structure de cette enzyme et le gène responsable du SHM (Hal) sont situés à deux locus distincts. Les deux lignées diffèrent par la valeur du déséquilibre de linkage entre Hal et PHI qui est très hautement significatif dans la lignée $\mathrm{A}$ et non significatif dans la lignée $\mathrm{B}$. La fréquence de l'allèle $\mathrm{Hal}^{8}$ responsable du SHM est significativement plus forte dans la lignée B que dans la lignée A en considérant une pénétrance de $\mathrm{Hal}^{8}$ égale à $\mathrm{o}, 75$, ce qui confirme $1^{\prime}$ hypothèse d'une association entre l'hypertrophie musculaire et le syndrome d'hyperthermie maligne. L'augmentation observée de la fréquence de l'allèle $\mathrm{PHI}^{\mathrm{B}}$ en lignée $\mathrm{B}$ par rapport à la lignée $\mathrm{A}$ est vraisemblablement due au déséquilibre existant entre les locus Hal et PHI et non à un effet propre du locus PHI sur le développement musculaire.
\end{abstract}

Le syndrome d'hyperthermie maligne chez le porc semble être gouverné par un gène autosomal récessif à pénétrance élevée (voir par exemple OLLIVIER et al., I975). Nous désignerons ce gène par $\mathrm{Hal}^{s}$ et l'allèle normal par $\mathrm{Hal}^{+}$. Une association entre la sensibilité des animaux à l'halothane, agent anesthésiant qui permet de détecter le syndrome, et le génotype aux locus de la phosphohexose isomérase 
(PHI) et du groupe sanguin $\mathrm{H}$ a été montrée par JøRGENSEN et al. (I977) chez les Landrace Danois, Néerlandais et Belge. Cette observation a été précisée par la mise en évidence d'une liaison étroite entre les locus Hal et PHI (ANDRESEN et JENSEN, I977). La liaison entre les locus de la PHI et de la 6-phosphogluconate déshydrogénase (6-PGD) a d'autre part été démontrée par ANDRESEN (I97I). Chez le Piétrain, Ansay et Olirvier (I978) ont mis en évidence un déséquilibre de linkage entre les locus Hal et PHI alors qu'il n'y a pas de déséquilibre entre les locus Hal et 6-PGD d'une part, PHI et 6-PGD d'autre part. L'étude des déséquilibres de linkage entre les locus Hal, PHI et 6-PGD a été poursuivie chez le Piétrain et les résultats présentés ici portent sur un échantillon de $25^{\circ}$ porcs, mâles et femelles, anesthésiés au poids vif de $20-25 \mathrm{~kg}$ à l'élevage d'Avord (Cher). Ces porcs proviennent de deux lignées $\mathrm{A}$ et $\mathrm{B}$ sélectionnées à partir d'une même population initiale depuis 3 générations, l'une (A) pour une plus forte vitesse de croissance et une moindre épaisseur de lard dorsal, et 1'autre (B) pour l'hypertrophie musculaire appréciée visuellement.

Le sang a été récolté à la veine cave immédiatement avant l'épreuve à l'halothane dont les modalités sont décrites par OLLIVIER et al. (I975). Les échantillons ont été prélevés en tubes vacutainer héparinés, lavés trois fois avec une solution de $\mathrm{NaCl} 0,9 \mathrm{p}$. roo. Les culots de globules rouges sont conservés à $-20^{\circ} \mathrm{C}$ jusqu'à leur utilisation. Les culots sont alors décongelés à la température du laboratoire, dilués deux fois avec de l'eau distillée et agités sur vortex pour provoquer l'hémolyse. Les hémolysats sont centrifugés pendant $30 \mathrm{mn}$ à I $500 \mathrm{~g}$ et la partie supérieure est utilisée pour l'électrophorèse.

Les échantillons ont été analysés par électrophorèse en gal d'amidon selon une méthode très voisine de celle de BENGTSSON et SANDBERG (I973). La dimension des plaques était de $23 \times 8 \times 0,6 \mathrm{~cm}$, la concentration en amidon de II p. IOO, la migration de $\mathrm{I} 8 \mathrm{~h}$ sous une tension de $6 \mathrm{~V} / \mathrm{cm}$ à $-+4^{\circ} \mathrm{C}$; enfin le tampon phosphate a été ajusté à $\mathrm{pH} 7,0$ avec $\mathrm{NaOH}$. Nous avons aussi utilisé 1'électrophorèse sur acétate de cellulose (Cellogel 5,7 $\times \mathrm{r}_{4} \mathrm{~cm}$, Chemetron, Italie), selon la technique de WidAr et al. (I975) avec un temps de migration de $\mathrm{I}$ h $30 \mathrm{mn}$ pour la PHI et de $30 \mathrm{mn}$ pour la 6-PGD. Dans les deux méthodes nous avons employé la coloration spécifique des deux enzymes selon Bengtsson et SANDBERG (I973).

Les déséquilibres de linkage entre les locus Hal, PHI et 6-PGD ont été estimés par les méthodes de HILL (I974), modifiées pour prendre en compte une valeur (supposée connue) de la pénétrance $(w)$ de l'allèle récessif au locus Hal. Les calculs ont été faits avec $w=\mathrm{I}$ et $w=0,75$.

Pour les tests statistiques, il a été ajouté aux variances d'échantillonnage que donnent les méthodes précédentes, des variances dues à la dérive génétique qui résulte de l'effectif limité des deux lignées. Pour un gène de fréquence $p$, on sait que la variance de dérive est égale à $\mathrm{F} p(\mathrm{I}-p), \mathrm{F}$ étant le coefficient de consanguinité. Les valeurs de $\mathrm{F}$ utilisées ici sont les accroissements de consanguinité calculés d'après les pedigrees dans les deux lignées au cours des 3 générations écoulées depuis le moment où elles ont été séparées, soit 0,03 dans la lignée A et 0,04 dans la lignée $B$. Les effets de la sélection sur la dérive des fréquences géniques ont été ignorés, car ils sont négligeables dans les premières générations de sélection (voir NICHOLAS et ROBERTSON, I976). Les effets de la dérive génétique sur le déséquilibre de linkage ont été étudiés par HIL, et RoBERTson (I968) qui donnent des expressions permettant de calculer la variance du déséquilibre $D$ à chaque génération en fonction de trois moments (impliquant $\mathrm{D}$ et les fréquences géniques) à la génération précédente et deux paramètres, l'effectif génétique $\mathrm{N}$ et le taux de recombinaison $c$. Les valeurs initiales de ces moments sont calculées à partir des 
valeurs de $\mathrm{D}$ et des fréquences géniques estimées globalement sur l'ensemble des deux lignées. Quant aux deux paramètres, 1'effectif génétique $\mathrm{N}$ est déduit de 1'accroissement de consanguinité calculé plus haut, soit $\mathrm{N}=50$ dans la lignée $\mathrm{A}$ et $N=38$ dans la lignée $B$, et les taux de recombinaison $c$ utilisés sont ceux proposés par ANDRESEN et JENSEN (I977), soit o entre Hal et PHI et o,06 entre PHI et 6-PGD. Les effets éventuels de la sélection sur D et sur sa variance de dérive ont été ignorés.

Les données du tableau I confirment celles d'ANSAY et OLLIVIER (I978) qui rapportaient l'existence, parmi 57 animaux sensibles à l'halothane, d'un sujet

\section{TABLEAU I}

Répartition des animaux dans les deux lignées selon leur sensibilité à l'halothane et leur phénotype pour la PHI et la 6-PGD

Distritution of the pigs in the two lines according to halothane sensitivity and phenotype for $P H I$ and 6-PGD

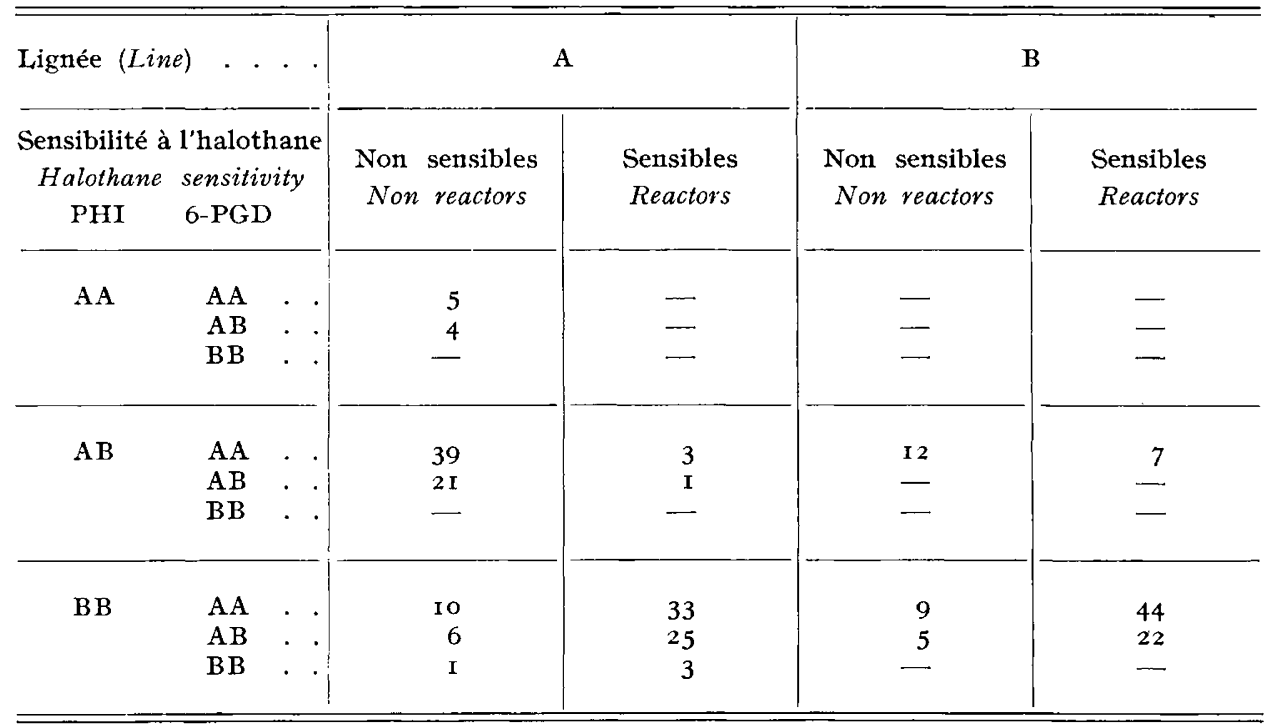

de phénotype PHI $A B$. Les onze animaux de ce type trouvés dans notre étude démontrent de manière formelle que le gène de structure de l'enzyme PHI et le gène responsable de la sensibilité à l'halothane sont situés à deux locus distincts.

Le déséquilibre de linkage (tabl, 2) calculé entre les trois locus PHI, 6-PGD, Hal pris deux à deux n'est significatif que pour le couple $\mathrm{PHI}-\mathrm{Hal}(\mathrm{P}<0,00 \mathrm{r})\left({ }^{1}\right)$ et seulement dáns la lignée $\mathrm{A}$. Il implique une fréquence des combinaisons alléliques $\mathrm{PHI}^{\mathrm{A}} \mathrm{Hal}^{s}$ et $\mathrm{PHI}^{\mathrm{B}} \mathrm{Hal}^{+}$inférieure à celle attendue ("répulsion ") et par conséquent une fréquence supérieure des combinaisons $\mathrm{PHI}^{\mathbf{B}} \mathrm{Hal}^{8}$ et $\mathrm{PHI}{ }^{\mathrm{A}} \mathrm{Hal}^{+}$("attraction »)

(I) La prise en compte de l'hypothèse de pénétrance incomplete $(w=0,75)$ dans la méthode d'estimation a peu d'effet sur le déséquilibre estimé mais accroît la variance de l'estimateur. 
TABLEAU 2

Fréquences géniques ( \pm écart-type)

et déséquilibres de linkage relatifs aux trois locus Hal, PHI et 6-PGD dans les deux lignées

Gene frequencies ( \pm standard error)

and linkage disequilibria relative to the three loci $\mathrm{Hal}, \mathrm{PHI}$ and 6-PGD in the two lines

\begin{tabular}{|c|c|c|c|}
\hline $\begin{array}{l}\text { Allèles et locus } \\
\text { Alleles and loci }\end{array}$ & $\begin{array}{l}\text { Lignée A } \\
A \text { line }\end{array}$ & $\begin{array}{l}\text { Lignée B } \\
B \text { line }\end{array}$ & $\begin{array}{c}\text { Test } t \text { des } \\
\text { différences entre } \\
\text { lignées (b) } \\
t \text { test for differences } \\
\text { between lines }\end{array}$ \\
\hline \multicolumn{4}{|l|}{$\begin{array}{l}\text { Fréquence génique (Gene fre- } \\
\text { quency): }\end{array}$} \\
\hline 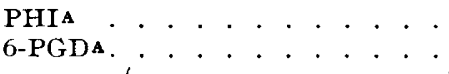 & $\begin{array}{l}0,272 \pm 0,08 \mathrm{I} \\
0,785 \pm 0,075\end{array}$ & $\begin{array}{l}0,096 \pm 0,063 \\
0,864 \pm 0,073\end{array}$ & $\stackrel{\S}{\text { NS }}$ \\
\hline $\mathrm{Hal}^{s}$ (a) $\left\{\begin{array}{l}w=\mathrm{I} \\
w=\mathrm{o} \\
w\end{array}\right.$ & $\begin{array}{l}0,656 \pm 0,088 \\
0,75^{8} \pm 0,082\end{array}$ & $\begin{array}{l}0,859 \pm 0,074 \\
0,992 \pm 0,035\end{array}$ & $\underset{\S}{\S}$ \\
\hline \multicolumn{4}{|l|}{$\begin{array}{l}\text { Déséquilibre de linkage (Linkage } \\
\text { disequilibrium): }\end{array}$} \\
\hline PHI et $6-\mathrm{PGD} . . .+. . .$. & 0,02 I NS & $0,013 \mathrm{NS}$ & NS \\
\hline PHI et $\mathrm{Hal} \quad\left\{\begin{array}{l}w=\mathrm{I} . . \\
w=0,75 .\end{array}\right.$ & 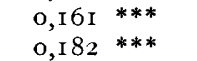 & $\begin{array}{l}0,044 \mathrm{NS} \\
0,044 \mathrm{NS}\end{array}$ & $\S$ \\
\hline 6-PGD et Hal $\left\{\begin{array}{l}w=\mathrm{I} . \\
w=0,75 .\end{array}\right.$ & $\begin{array}{l}0,025 \mathrm{NS} \\
0,029 \mathrm{NS}\end{array}$ & $\begin{array}{ll}0,014 & \text { NS } \\
0,002 & \text { NS }\end{array}$ & $\begin{array}{l}\text { NS } \\
\text { NS }\end{array}$ \\
\hline
\end{tabular}

(a) $w$ : Pénétrance de l'allèle $\mathrm{Hal}^{s}$ (Penetrance of the Hals allele).

(b) NS : Non significatif (P>0,Io) (Not significant) $(P>0 . I o)$.

$$
\begin{array}{ll}
\S & \mathrm{P}<0,10 . \\
* & \mathrm{P}<0,05 . \\
* * & \mathrm{P}<0,01 . \\
* * * & \mathrm{P}<0,001 .
\end{array}
$$

qui explique l'association entre le génotype de la PHI et la sensibilité à l'halothane, déjà observée par JøRGENSEN et al. (I977).

L'examen đu tableau 2 confirme la prédominance des allèles $\mathrm{PHI}{ }^{\mathrm{B}}$ et $6-\mathrm{PDG}^{\mathrm{A}}$ dans la race de Piétrain (ANsAy et OLLIVIER, I978). De plus on observe une fréquence de 1'allèle $\mathrm{Ha}^{s}$ plus élevée dans la lignée $\mathrm{B}$ que dans la lignée A : la différence est significative au seuil de I p. Ioo pour $w=0,75$ et atteint le seuil de ro $\mathrm{p}$. Ioo pour $w=\mathrm{I}$. La fréquence des animaux sensibles à l'halothane passe de $43 \mathrm{p}$. Ioo en lignée $\mathrm{A}$ à 73 p. Ioo dans la lignée B; il est tentant de penser que cette augmentation est due à la sélection en faveur d'un très fort développement musculaire dans la lignée B, selon 1'hypothèse, avancée par OLLIVIER et al. (I975), d'une association positive entre le syndrome d'hyperthermie maligne et l'hypertrophie musculaire.

Une augmentation significative au seuil de ro p. Ioo, de la fréquence de l'allèle $\mathrm{PHI}^{\mathrm{B}}$ est observée en lignée $\mathrm{B}$ par rapport à la lignée $\mathrm{A}$. Elle estvraisemblablement due au déséquilibre de linkage qui tend à associer cet allèle à l'allèle $\mathrm{Hal}^{s}$ et non à un effet propre du locus PHI sur le développement musculaire, hypothèse en accord avec l'étude de ANSAy et Olifivier (I978) sur le taux de créatinine plasmatique.

Il semble, à ce stade, nécessaire de préciser la position du locus Hal par une 
étude de la ségrégation simultanée des allèles aux locus Ha1, PHI, 6-PGD chez les descendants d'animaux hétérozygotes, et de clarifier les incidences sur ces trois locus du mode de sélection appliqué dans les deux lignées.

Reçu pour publication en avril 1978.

\section{Remerciements}

Nous remercions D. TANGUy (Génétique animale, Jouy-en-Josas) pour la mise au point des programmes de calculs.

\section{Summary \\ Linkage disequilibria between the $\mathrm{Hal}$ (malignant hyperthermia), PHI and 6-PGD loci in two Pietrain lines}

Two hundred and fifty Pietrain pigs from two lines selected for 3 generations, one (A line) on a combination of average daily gain and backfat thickness and the other (B line) on muscular hypertrophy, were submitted to a halothane-test and their genotypes determined for two red cell enzymes (PHI and 6-PGD). The occurrence in the halothane sensitive group of II animals heterozygous at the PHI locus definitely proves that the structural gene of this enzyme and the gene controlling MHS (Hal) are located at two distinct loci. The two lines differ with respect to the linkage disequilibrium between $\mathrm{Hal}$ and $\mathrm{PHI}$, which is very highly significant in the $\mathrm{A}$ line and not significant in the $\mathrm{B}$ line. The frequency of the $\mathrm{Hal}^{s}$ allele responsible for SHM is significantly higher in the $\mathrm{B}$ line than in the $\mathrm{A}$ line, assuming a penetrance of $\mathrm{Hal}^{8}$ equal to 0.75 , which is in favour of the hypothesis of an association between muscular hypertrophy and malignant hyperthermia syndrome. Moreover, the higher frequency of the PHIB allele observed in the B line compared to the $\mathrm{A}$ line is probably due to the linkage disequilibrium found between the $\mathrm{Hal}$ and PHI loci, and not to a real effect of the PHI locus on muscular development.

\section{Références bibliographiques}

ANDRESEN E., I97I. Linear sequence of the autosomal loci PHI, $\mathrm{H}$ and 6-PGD in pigs. Anim. Blood Grps biochem. Genet., 2, I19-I 20.

ANDRESEN E., JENSEN P., I977. Close linkage established between the Hal locus for halothane sensitivity and the PHI locus in pigs of the Danish Landrace breed. Nord. Vet. Med., 29, 502-504.

ANSAy M., OLLIVIER L., I978. Créatinine plasmatique et sensibilité du porc au syndrome d'hyperthermie maligne. Relations avec deux enzymes du globule rouge (PHI et 6-PGD). Ann. Génét. Sél. anim., 10, 9-16.

BENGTSSON S., SANDBERG K., I973. A method for simultaneous electrophoresis of four horse red cell enzyme systems. Anim. Blood Grps biochem. Genet., 4, 83-87.

HILL W. G., I974. Estimation of linkage disequilibrium in randomly mating populations. Hevedity, 33, 229-239.

HILL W. G., Robertson A., I968. Linkage disequilibrium in finite populations. Theor. Appl. Genet., 38, 226-23I.

Jørgensen P. F., Hyldgaard-Jensen J., Eikelennboom G., Moustgaard J., 1977. Meat quality, halothane sensitivity and blood parameters. In Proceedings $3^{\text {rd }}$ intern. Conf. on Production Disease in Farm Animals, 200-zoz, Pudoc, Wageningen.

Nicholas F. W., ROBERTSON A., 1976. The effect of selection on the standardized variance of gene frequency. Theor. Appl. Genet., 48, 263-z68.

OLLIVIER L., SELLIER P., MONIN G., 1975. Déterminisme génétique du syndrome d'hyperthermie maligne chez le porc de Piétrain. Ann. Génét. Sél. anim., 7, 159-166. 